\title{
Utilizing Principles of Clinical Hypnosis for an Effective Communication with Patients in Managing Chronic Pain
}

\author{
Dr Anand Chandrasegaran ${ }^{1^{\star}}$, Manjit Singh $^{1}$ \\ ${ }^{1}$ Columbia Asia Hospital - Klang, Selangor, Malaysia
}

This report is an undertaking to illustrate the application of hypnosis in the initial patient doctor consultation to build better patient rapport. An effective communication in consultation leads to better rapport with the patient for a comprehensive chronic pain management. This case involves a young patient who presented with worsening body ache and insomnia. She sought consultations and treatment from 6 doctors. She was not satisfied with these consultations. All investigations showed negative clinical findings with same diagnosis from all doctors. She was seen in the author's pain clinic, for chronic pain management. Hypnotic principles were used to manage the consultations and as therapeutic option for her problems. In the initial consultation, the author used Rogerian approach in-cooperating hypnosis in establishing empathy with the patient. This was followed by formal hypnotic intervention as a therapeutic intervention. Patient's expectation was met and solved her initial presenting medical problem, which the patient was not able to achieve with previous consultations with 6 different doctors. Better management of patient's presenting complain should take into account the perceptions and interactions of patients and physicians. Physicians need to empathize, listen, elicit patients' beliefs and expectations, and offer a range of treatments, including cognitive and behavioural therapies, tailored to individual needs. In this strategy, communication could be improved by applying basic principles of hypnotherapy. Doctor's dominance in relation to the patient-professional communication need to be reexamined.
\end{abstract}

Keywords: Effective communications, clinical hypnosis, chronic pain management

\section{INTRODUCTION}

This paper is an effort to advocate a constructive model highlighting the utilisation of principles of hypnosis techniques, in the initial patient-doctor consultation to establish stronger patient rapport, establishing empathy with the patient and how these contributed to managing

\footnotetext{
*Correspondence: mbbsumagmail.com

Dr Anand Chandrasegaran, Columbia Asia Hospital - Klang , Selangor, Malaysia
}

Received: 06 March 2018 Accepted: 26 April 2018

Sleep and Hypnosis

Journal homepage:

www.sleepandhypnosis.org

ISSN: 2458-9101 (Online) the patient's presenting symptoms( chronic paingeneralized body ache). This was a subject who had sought consultations from six doctors, with no concrete therapeutic outcome to the patient's presenting symptoms. This patient was reviewed at the author's pain management clinic. The patient's medical issues were managed with hypnotic intervention. We would like to highlight how anaesthetist could establish an empathy with the patient for a dynamic and comprehensive chronic pain management care for patients. Patients with chronic pain often don't get proper care and treatment, as there is always an psychological componenet involved in their disease process. Some physicians failed to establish empathy with patients due to lacking of knowledge on its importance and techniques involve. 
Communication skills is unquestionably a vital ability in current healthcare practice (Lipkin, Quill, \& Napodano, 1984). This delicate skill in not being incorporated with considerable scrutiny into most conventional medical teaching. In traditional anaesthesia practice, we anaesthetist have not been brought to light to the gravity of establishing strong rapport during consultation with patients. Much valuable diagnostic data can be obtained from the initial history taking from patients. The physician's interpersonal skills plays a decisive part in influencing the patient's gratification and conformity to proposed treatment (Roter, 1977).

"Hypnosis" refers to a modified conscioiusness and the conduct by which this state is induced (Turner \& Chapman, 1982). During hypnosis the physiology, cognitive, and affective processes as well as behaviour can be influenced positively. This phenomena can be brought about by another individual (hypnotism) or by patients themselves (self-hypnosis). The subjective experience of hypnosis is described by a tremendous degree of feeling as convincing and "it takes effect by itself". Hypnosis is not authoritarian, passive, and focused around the therapist/ doctor (Peter, 2015). It is a support and solution oriented therapy in which the focus is on the patient's own positive potentials (Turner \& Chapman, 1982). The notion of hypnosis as an authoritarian, manipulative practice is owing to its usage in stage appearances. This is the toughest hindrance to the re-assimilation of hypnosis into current medical practice.

Here, the writer adopted hypnotic techniques for an effective communication and as a therapeutic means in managing the patient's chronic pain (generalised unspecific body ache). Cognitive behavioural approach and hypnotherapy as a beneficial adjuct in chronic pain management, has been extensively studied (Wright, 1977).

\section{CASE REPORT}

The patient being descripted in this case has consented (written consent given) for the case to be discussed and shared as clinical case study. This is a case of a 30-year- old female with one young child. She works as an advertising executive. Her passion is to work as a nutritionist (she completed her undergraduate studies in nutrition); but she couldn't get appropriate job to suite her passion. There was an element of career frustration to her present position, which was contributing to some amount of stress to her.

She has been suffering with generalized muscular pain especially more on her left shoulder region for 4 months. The quality of this pain was unspecific. The pain was characterised as dull in nature and present on most of the time. This pain was not correlated with any significant postural variations. No significant neurological deficit associated with this pain. There was no record of trauma that could explain the cause of this patient's non-specific muscular pain. Ever since she started having this muscular ache, patient developed sleeping difficulties (insomnia). Between these two symptoms, she reported that the insomnia was more disturbing compared to her complain of generalised body ache.

She has sought treatment from three doctors at general practitioner facilities and underwent extensive medical test and radiological assessment at three different clinics since her illness started. All of them offered her the same mode of treatment. They advised her to have adequate rest and diagnosed her symptoms attributed to her stressful work life. There was no clinical evidence of pathology that was contributing to the unspecific generalised body myalgia. However, she was not satisfied with the doctors' explanations and treatment offered. She got a review from an Orthopaedic consultant. The doctor did extensive blood investigation and MRI investigation of her left shoulder and her spine. There were no obvious pathology detected from these investigations. The orthopaedic doctor, concluded that she could be exhibiting a situation called, psychosomatic disorder (Kirschenbaum \& Jourdan, 2005) and the patient would benefit from a psychiatric intervention. She was referred for a psychiatric evaluation for further management.

She visited the psychiatric consultant and had the necessary assessment. He prescribed her anxiolytic medication and an oral analgesia, to treat her symptoms. She was strictly told to adhere to the drug regime, even though these medications may have some undesireable effects (which was said to be mild and self limiting side 
effects such as drowsiness). She was told that, being compliant to the medications was essential to treat her insomnia problem. No definite diagnosis was given to the patient concerning her insomnia and unspecific generalised body myalgia.

She was not satisfied with these consultations (the orthopaedic and psychiatric evaluation). She mentioned that they both didn't devote time with her in analysing her core issues and took time to clarify what's amiss with her. None of them could meet her expectation of a holistic physician. What upset her most was, she got the impression that they were all brushing her symptoms aside, as though she was suffering form a psychological disorder. No precise information offered to her symptoms. The patient believes she has a medical condition that demands urgent scrutiny. She was not compliant with the medications as they made her drowsy (undesireable side effects of the medications) at work time and she could not go on her routine daily task.

Since taking the anxiolytic medications, her insomnia problem worsens, and she started to develop palpitations. She decided to have an assessment by a cardiologist with regard to her palpitation symptom. Extensive investigation by the cardiologist showed no anomalies in her cardiac function. Hormonal blood investigation and other relevant blood studies with regard to the patient's symptom of generalised body aches and palpitation, were done. All results were normal. She was offered an orthopaedic review, but she rejected, as she said, she previously had orthopaedic assessment just about a month ago. She was offered for assessment with the anesthesiology (the author) for chronic pain management (for her symptom of unspecific generalised body myalgia). Patient agreed for an appointment and further care.

\section{Intervention and Treatment Strategy}

Patient was met in the author's pain clinic. The author approached the patient's consultation with Rogerian's (Liossi \& Hatira, 1999). Approach supported by cognitive behaviour (Castel, Salvat, Sala, \& Rull, 2009; Hartland, 1971). Centred approach, to get further history and weigh patient's believe of her illness. The steps involved were (Lang et al., 2000; Lang et al., 2006; Lang et al., 2008):
1. Establishing a therapeutic alliance with the patient

2. Identifying the patient's major issues $\&$ goals

3. Creating positive expectancies

4. Creating various hypnotic phenomena by hypnosis techniques

Patient was allowed to describe her symptoms at her own pace and directions. Patient's initial history taking was probing into her insomnia problem and the generalised body aches. Five minutes into the interview, patient was more comfortable and appeared more relaxed. She started to concede more medical and social history. She was a well and active person prior to this. Last 5 months, she had difficulty to sleep at night despite being tired. These symptoms started owing to her work pressure. As the problem worsen, she was worried that her sympotoms could be signs of a more sinister clinical diagnosis such as malignancy. As her insomnia worsens, her body aches worsened. This made her more disturbed and created a negative vicious cycle. As her insomnia worsen, her concern that "something was amiss" with her health, worsen too. Every doctor she met didn't show the empathy she was expecting. When she met the psychiatrist doctor, she was upset with his way of handling her, because, he didn't give the patient space to acknowledge her emotions. Her expectation was, as a psychiatrist, the doctor could take up the role of being her counselor, and listen to her problems in an empathic manner.

Once patient divulged more of her concerns, she was more relaxed with the writer's approach and a cordial rapport was formed immediately. The patient acknowledged that worry over her symptoms made them worse, created a vicious circle. Patient was offered formal hypnotic intervention for pain management with hypnosis (Castel et al., 2009), intending at providing a solution to the patient's malady. The author further proposed at teaching the patient self-hypnosis to scale down her body aches and insomnia problems,with practices at home. Patient was briefed on relaxation hypnosis process.

Myths about hypnotherapy were explored, debated, and straightened out. Examples of frequently accepted techniques to mitigate and deal with symptoms, e.g., hand warmth on the affected part of her body and imagery 
demonstrated. Content and procedure of hypnotherapy and its mechanisms and expected results were pointed out. Questions were answered and consent form was completed. As part of managing the patient's chronic pain, hypnotherapy sessions for pain management (Bikker, Mercer, \& Reilly, 2005) as a treatment module, was explained to this patient. The purpose in this discussion was to allow the patient to become acquainted with hypnosis and the therapy setting. The patient also received information about insomnia including its pathophysiology, symptoms, treatments, and the contribution of psychological factors. The patient was suggested to practice similar relaxation a home. During her self hypnosis exercise, the patient was emphasised that it is her time to relax as her body eases all the pain and aches by naturally relaxation. This learning skills will improve with practice and that she was required to do this everyday. Since therapy was not a passive process, her active involvement was paramount.

Standard protocol of hypnotic relaxation with deep breathing was done (Hartland, 1971). The focus was to relax the physical body, by Hartland's (Hartland, 1971). Progressive muscle relaxation (muscle relaxation visualization from the top of head to the toes). We used Eriksonian hypnotic induction approach. In this hypnotic state, we included ego-strengthening and visualization of a well rested person, and healing imaginary. This session took us about 30 minutes.

Patient reported as feeling energised and verbalised that she felt a sense of self satisfying. She managed to revealed her concerns and anxiety, which she never did with earlier doctors.

Patient scheduled her next appointment, 2 weeks later. At that session, patient's self - hypnosis techniques were reviwed. A combination of direct and permissive hypnotic suggestions were used. Since this was her second session, she was more receptive to hypnosis suggestion now. In this session, we approach the patient with similar Rogerian Approach, and use CB (cognitive behavioural) skills. Which was followed by formal hypnosis for relaxation, and ego strengthening and we included metaphors of being in self control of her situations all the time.

Her insomnia and body ache problems resolved almost $80 \%$. She kept a diary of her sleeping pattern. We discussed on the diary. After the first hypnotic session,the patient reported as being able to have more quality of restful sleep. She was not taking any pain medications for her body aches anymore.

Patient was follow-up on 2 months after the last session. She reported her insomnia issue resolved. She prefers this intervention as it doesn't need additional medications or any invasive procedures. She was not comfortable to take anxiolytic medications, as prescribed by her initial psychiatrist, because it gave a negative stigma to her (made her think she has mental illness). The days she can't have proper quality sleep, she uses the self hypnosis skills. She is more productive at office after the discussions, and able to do her regular task as what she used to do.

\section{Discussion points}

When she met the author in the hospital, the Rogerian approach (Liossi \& Hatira, 1999), gave space for the patient to be relaxed and ample of time to reveal her view of the history. The 3 core aspects used her to create good quality therapeutic alliance were

1. Congruence of the author to relate to the patient with genuineness

2. Unconditional positive regard to patient's presenting history

3. Empaty to experience the patient from her perspective

She was satisfied with the author's consultation. Reason being, in the Rogerian approach, the patient viewed at an empathy angle and no bias judgments formed during the initial conversations with the patient. Conversational hypnosis (Lipkin et al., 1984). Techniques, proved valuable in this case. Hypnosis can be started with or without formal induction procedure. The hypnotic state is part of everyday existence (Turner \& Chapman, 1982).

This allowed the patient to share her thoughts with the doctor, who is not controlling the flow of the discussion with minimal interruption from the physician and being attentive to the patient's expectation (Bartlett et al., 1984). The author, allowed the patient to interpret her reasons for the symptoms and use of CB skills, allowing 
the patient to analyse her symptoms. Instead of the traditional approach whereby the physician interprets the symptoms and directing the flow of the consultation, the author made a treatment strategy with the patient (active involvement from patient instead the conventional approach of passive involvement).

Evidence has demonstrated that issues in doctorpatient communication are frequent and influence patient management (Dyas et al., 2010). Active listening to patients' concerns is the trait of a physician most craved by patients (Dyas et al., 2010). Poor clinical communication can contribute to increasing patient frustration with the medical consultation and failure to manage chronic pain . In many healthcare institutions serious communication problems are prevalent and this prevents comprehensive chronic pain management.

To become competent clinical communicators, physicians must grasp a defined body of knowledge, skills, and attitudes. Patient centred consultation (Rogerian approach) gave autonomy to the patient to analyse issues at their own pace. In the doctor centred consultations the doctor is right, busy clinic practice, and the brief consultation time, doesn't favour patient's need (Bingel, 2014), as detailed by the patient in her earlier consultations with 6 different doctors before coming to the author's clinic.

Rogerian approach with conversation hypnotic skills, favors rapid rapport establishment. rapport is imperative to have an effective consultation and to set up a thorough medical treatment plan with patient's goal (medically realistic) in consideration. An empathic physician who forms good rapport, and makes constructive suggestions, about an upcoming medical procedure / consultation and treatment strategy, can aid in reducing the patient's physical and emotional stress from the first consultations.

In this patient, the author used hypnotic techniques for:

1. Hypnotic communication- Waking suggestions (suggestions without trance induction): for detailed history taking.

2. Hypnotherapy (psychotherapy while the patient under hypnosis) : problem management by giving the patient access to their own resources
Better management of insomnia should take into account the perceptions and interactions of patients and physicians. Physicians need to empathise, listen, elicit patients' beliefs and expectations, and offer a range of treatments, including cognitive and behavioural therapies (adapted to individual demands). Physician's treatment plan should assimilate an awareness of patients' decisionmaking processes, the physicians' role during the consultation, and how to negotiate and deliver strategies for resolving sleep problems (Hauser, Hansen, \& Enck, 2012).

Together with legitimate and compassionate communication (Hansen \& Bejenke, 2010), effective doctor-patient communication has to consist of:

1. Restraint of negative suggestions (Lamberg, 1999; Seemann, Zech, Graf, \& Hansen, 2015).

2. Use of constructive suggestions (Lamberg, 1999).

In this strategy, communication could be enhanced by applying basic principles of hypnotherapy and gradually replacing physician's dominance in relation to the patientdoctor professional relationship (Brugel, PostmaNilsenova, \& Tates, 2015). In 2015, the German Medical Assembly, supports initiatives to strengthening physician's communicative competence (Hauser et al., 2012). Controlled experimental studies are needed to establish the efficacy of hypnosis in having effective communitions in patient's with chronic pain.

Acknowledgment: We would like to thanks Ms Sheila Menon and Dr Alan Soh of London College of Clinical Hypnosis (LCCH ASIA) in guiding us and sharing their experiences in developing us. We are humbly grateful to the patient for her active participation in the clinical process.

Conflict of interest: The authors declare no conflict of interest.

Informed consent: Informed consent was obtained from all the participants included in the study.

Funding: There are no funding from external sources involved in this presentation. 


\section{References}

Bartlett, E. E., Grayson, M., Barker, R., Levine, D. M., Golden, A., \& Libber, S. (1984). The effects of physician communications skills on patient satisfaction - recall, and adherence. Journal of Chronic Diseases, 37(9-10), 755-764. doi:Doi 10.1016/00219681(84)90044-4

Bikker, A. P., Mercer, S. W., \& Reilly, D. (2005). A pilot prospective study on the consultation and relational empathy, patient enablement, and health changes over 12 months in patients going to the Glasgow homoeopathic hospital. Journal of Alternative and Complementary Medicine, 11(4), 591-600. doi:DOI 10.1089/acm.2005.11.591

Bingel, U. (2014). Avoiding nocebo effects to optimize treatment outcome. Jama-Journal of the American Medical Association, 312(7), 693-694. doi:10.1001/jama.2014.8342

Brugel, S., Postma-Nilsenova, M., \& Tates, K. (2015). The link between perception of clinical empathy and nonverbal behavior: The effect of a doctor's gaze and body orientation. Patient Education and Counseling, 98(10), 1260-1265. doi:10.1016/j.pec.2015.08.007

Castel, A., Salvat, M., Sala, J., \& Rull, M. (2009). Cognitivebehavioural group treatment with hypnosis: a randomized pilot trail in fibromyalgia. Contemporary Hypnosis, 26(1), 48-59.

Dyas, J. V., Apekey, T. A., Tilling, M., Orner, R., Middleton, H., \& Siriwardena, A. N. (2010). Patients' and clinicians' experiences of consultations in primary care for sleep problems and insomnia: a focus group study. British Journal of General Practice, 60(574). doi:10.3399/bjgp10X484183

Hansen, E., \& Bejenke, C. (2010). Negative and positive suggestions in anaesthesia - Improved communication with anxious surgical patients. Anaesthesist, 59(3), 199-+. doi:10.1007/ s00101-010-1679-9

Hartland, J. (1971). Further observations on the use of "egostrengthening" techniques. American Journal of Clinical Hypnosis, 14(1), 1-8.

Hauser, W., Hansen, E., \& Enck, P. (2012). Nocebo phenomena in medicine their relevance in everyday clinical practice. Deutsches Arzteblatt International, 109(26), 459-U426. doi:10.3238/arztebl.2012.0459

Kirschenbaum, H., \& Jourdan, A. (2005). The current status of Carl Rogers and the person-centered approach. Psychotherapy, 42(1), 37-51. doi:10.1037/0033-3204.42.1.37

Lamberg, L. (1999). Chronic pain linked with poor sleep; Exploration of causes and treatment. Jama-Journal of the American Medical Association, 281(8), 691-692. doi:DOI 10.1001/jama.281.8.691
Lang, E. V., Benotsch, E. G., Fick, L. J., Lutgendorf, S., Berbaum, M. L., Berbaum, K. S., ... Spiegel, D. (2000). Adjunctive nonpharmacological analgesia for invasive medical procedures: a randomised trial. Lancet, 355(9214), 1486-1490. doi:Doi 10.1016/S0140-6736(00)02162-0

Lang, E. V., Berbaum, K. S., Faintuch, S., Hatsiopoulou, O., Halsey, N., Li, X. Y., . . . Baum, J. (2006). Adjunctive self-hypnotic relaxation for outpatient medical procedures: A prospective randomized trial with women undergoing large core breast biopsy. Pain, 126(1-3), 155-164. doi:10.1016/j.pain.2006.06.035

Lang, E. V., Berbaum, K. S., Pauker, S. G., Faintuch, S., Salazar, G. M., Lutgendorf, S., . . Spiegel, D. (2008). Beneficial effects of hypnosis and adverse effects of empathic attention during percutaneous tumor treatment: When being nice does not suffice. Journal of Vascular and Interventional Radiology, 19(6), 897-905. doi:10.1016/j.jvir.2008.01.027

Liossi, C., \& Hatira, P. (1999). Clinical hypnosis versus cognitive behavioral training for pain management with pediatric cancer patients undergoing bone marrow aspirations. International Journal of Clinical and Experimental Hypnosis, 47(2), 104-116. doi:Doi 10.1080/00207149908410025

Lipkin, M., Quill, T. E., \& Napodano, R. J. (1984). The medical interview - a core curriculum for residencies in internal medicine. Annals of Internal Medicine, 100(2), 277-284. doi:10.7326/0003-4819-100-2-277

Peter, B. (2015). Hypnosis. In J. D. Wright (Ed.), International Encyclopedia of the Social \& Behavioral Sciences (2nd Edition ed., pp. 458-464). Oxford: Elsevier.

Roter, D. L. (1977). Patient participation in the patient-provider interaction: the effects of patient question asking on the quality of interaction, satisfaction and compliance. Health Education Monographs, 5(4), 281-315.

Seemann, M., Zech, N., Graf, B. M., \& Hansen, E. (2015). The premedication visit--suggestions for a patient-friendly design. Anasthesiol Intensivmed Notfallmed Schmerzther, 50(2), 142146. doi:10.1055/s-0040-100082

Turner, J. A., \& Chapman, C. R. (1982). Psychological interventions for chronic pain - a critical-review .2. operant-conditioning, hypnosis, and cognitive-behavioral therapy. Pain, 12(1), 23-46. doi:Doi 10.1016/0304-3959(82)90168-3

Wright, L. (1977). Conceptualizing and defining psychosomatic disorders. American Psychologist, 32(8), 625-628. 